\title{
Discriminant analysis of dual-wavelength spectral images for classifying poultry carcasses
}

\author{
Bosoon Park ${ }^{a, *}$, Kurt C. Lawrence ${ }^{a}$, William R. Windham ${ }^{\text {a }}$, \\ Yud-Ren Chen ${ }^{\mathrm{b}}$, Kevin Chao ${ }^{\mathrm{b}}$ \\ ${ }^{a}$ USDA, Agricultural Research Service, Richard B. Russell Research Center, P.O. Box 5677, Athens, \\ GA 30604-5677, USA \\ ${ }^{\mathrm{b}}$ Instrumentation and Sensing Laboratory, Beltsville, MD 20705-2350, USA
}

Received 17 January 2000; received in revised form 14 January 2002; accepted 10 February 2002

\begin{abstract}
An analysis of texture features, based on co-occurrence matrices (COMs), was conducted to determine the performance of dual-wavelength imaging for discriminating unwholesome poultry carcasses from wholesome carcasses. The variance, sum average, sum variance, and sum entropy of COMs were the most significant texture features $(P<0.005)$ for identifying unwholesome poultry carcasses. However, the feature values of angular second moment, variance, sum average, sum variance, and sum entropy did not vary with the COM parameters, distance and direction. The characteristics of variance and sum variance texture features varied with the wavelength of spectral images and with condemnation of poultry carcasses, as well. The sum variance of wholesome carcasses was higher $(P<0.005)$ than unwholesome carcasses for spectral images at $542 \mathrm{~nm}$. For 542 and $700 \mathrm{~nm}$ images, linear discriminant models were able to identify unwholesome carcasses with a classification accuracy of $91.4 \%$. However, a single linear discriminant model was not acceptable for identifying three different types of carcasses (wholesome, septicemic and cadaver), because of extreme inaccuracy for septicemic carcasses. In this case, the classifier that demonstrated the highest accuracy was $89.6 \%$ accurate at $542 \mathrm{~nm}$. Thus, a dual-wavelength imaging system with optical filters of 542 and $700 \mathrm{~nm}$ wavelengths appears promising for detecting unwholesome poultry carcasses. Published by Elsevier Science B.V.
\end{abstract}

Keywords: Image processing; Poultry inspection; Machine vision; Automation

* Corresponding author. Tel.: + 1-706-546-3396; fax: + 1-706-546-3633.

E-mail address: bpark@saa.ars.usda.gov (B. Park). 


\section{Introduction}

Poultry carcass inspection is mandated by the Food Safety Inspection Service (FSIS). Since the majority of inspection tasks are highly repetitive, extremely boring, and their effectiveness depends on the efficiency of human inspectors, machine vision is a technology well suited for providing automated production processes with vision capabilities. Even though machine vision has evolved into promising technology for agricultural product applications including grading, inspection, identification, or classification (Paliwal et al., 1999; Luo et al., 1999; Majumdar and Jayas, 1999; Wang et al., 1999; Giacomelli et al., 1998; Pearson and Schatzki, 1998; Zayas and Flinn, 1998; Ni et al., 1998; Tao et al., 1995; Daley et al., 1994), there are still many factors, such as size, dimension, orientation, shape, color, and texture to be considered for application in poultry safety inspection.

In industrial situations, inspection is conducted by measurement and comparison of spatial geometry with known patterns, i.e. spatial pattern-recognition (Guyer et al., 1986). While spatial imaging resolves objects into their morphological dimensions, spectral imaging resolves a phenomenon that is the interaction of light with objects under inspection (Meyer et al., 1992). In general, a multispectral image provides more spectral characteristics of biological materials than a standard RGB color image, because multispectral images have the characteristics of a narrow-band spectral image, while RGB color images have broadband (normally 400-700 nm) information and are more difficult to understand spectrally. Multispectral imaging provides information about a material from variations in its spectral reflectance. In this case, the surface texture, reflectance intensity, size, and shape of the sample can be measured at specific wavelengths.

Since spectral imaging involves measuring the intensity of diffusely reflected light from the surface of an object, the reflected light contains information about the absorbers near the surface of the material. By using wavelengths selected across wavelength bands, it is possible to construct the characteristics of spectral features for a material.

Image texture has been used in image analysis for segmentation and classification for several years. Early studies of image texture analysis have involved autocorrelation functions (Liu et al., 1993), power spectra, and relative frequencies of various grey levels on unnormalized images (Park and Chen, 1994). Grey-tone spatial-dependence matrices, or co-occurrence matrices (COMs), also have been used for image texture analysis in agricultural applications (Shearer and Holmes, 1990; Park et al., 1992).

Since image texture contains statistical information of a grey-level image in the spatial domain, textural analysis may be able to classify images of condemned poultry carcasses. Also, image texture analysis is useful as a spectral image analysis tool because texture is independent of image tone. Thus, image texture analysis is an important process in image analysis because it partitions an image into meaningful separable categories, which could be employed to detect surface defects on poultry carcasses. However, there has been little research on model development for analyzing accuracy and performance of COMs as a function of parameters, such as angle and distance (Park and Chen, 1996), particularly for food safety inspection. 
The primary goals of this study were (1) to examine the performance of the co-occurrence matrix texture analysis method as a tool for dual-wavelength spectral image analysis for detecting condemned poultry carcasses, and (2) to develop linear and/or nonlinear discriminant models for classifying wholesome and unwholesome poultry carcasses.

\section{Materials and methods}

A total of 176 chicken carcasses, 73 wholesome and 103 unwholesome (30 septicemic, 48 cadaver, 16 tumor, five skin-tear, and four bruise), were used for collecting multispectral image data. A USDA veterinarian separated the carcasses based on the condition of condemnation. All samples were placed in plastic bags to minimize dehydration. The bags were then placed in coolers filled with ice and transported to the Instrumentation and Sensing Laboratory, Beltsville, Maryland to perform the multispectral image acquisition.

\subsection{Multispectral imaging system}

With an intensified multispectral camera (Model IMC-201, Xybion Electronic System, San Diego, CA) ${ }^{1}$, we used six interference filters with visible/near-infrared wavelengths of 542, 570, 641, 700, 720, and $847 \mathrm{~nm}$. Each filter has a bandwidth of $10 \mathrm{~nm}$. The camera has two on-board Motorola MC68HC11 microprocessors and a Sony XC-77 CCD imager with a $400-1000 \mathrm{~nm}$ spectral range (786 horizontal pixels by 493 vertical pixels spatial resolution). The imager was fiber-optically coupled to a microchannel plate intensifier. A Tokina AT-X lens with a variable focal length of $28-85 \mathrm{~mm}$ was used. The system's computer contained an 80486 Intel CPU with a $50 \mathrm{MHz}$ clock speed and an 8-bit image digitizer (ImCap, Xybion Electronic System, San Diego, CA). Two diffused high-intensity halogen lamps (Tota T-110, Lowel-Light, Inc., Brooklyn, NY) were used as the light source to provide at least 248 lux (lumen $/ \mathrm{m}^{2}$ ) of light on the chicken surfaces. A DC power supply (Nobatron DCR 150-5A, Raytheon Co., South Norwalk, CT) was used for regulating lamp power to minimize any variation in illumination excitation intensity. Spectral images were captured using commercial software (XICAS) and further processed and analyzed with in-house developed software. For this study, 438 wholesome, 180 septicemic, 288 cadaver, 96 tumor, 30 skin-tear, and 24 bruise spectral images (for a total of 1056 images, six different spectral images from each carcass) were acquired from the chicken carcasses sampled from poultry slaughterhouses.

\footnotetext{
${ }^{1}$ Mention of any company or trade name is for identification only and does not imply endorsement by the US Department of Agriculture.
} 


\subsection{Procedure}

A commercial shackle, attached to a stainless steel supporting rod, was used to hang individual chicken carcasses to acquire multispectral images. Two tungsten halogen lamps projected diffused light on the carcasses and were positioned to minimize glossy reflected light spots from the carcass surfaces. Six images were acquired from each carcass, one image at each wavelength specified in the previous section.

To improve image quality, system calibration was conducted prior to image acquisition to minimize the variation of each component of the system, such as illuminator power, filter characteristics, sample reflectance, and detector sensitivity. A $99 \%$ reflectance white Teflon ${ }^{\mathrm{TM}}$ panel was used as a reference for calibration of the imaging system because of its flat spectral reflectivity across the entire spectrum of interest. To acquire high-quality images and to prevent over-saturation of images at the 641-nm wavelength band, the lens aperture was set at $\mathrm{f} / 8$ and the gain of the intensifier was $70 \%$ of the maximum gain.

Since the $641 \mathrm{~nm}$ wavelength images had high intensity contrast with the background, they were used as templates for segmentation of each object (Park et al., 1996). Each segmentation template was then applied to other wavelength images to identify the carcass object in each image. All pixels of each object were digitized $(0-255)$ for the calculation of grey values. To obtain image texture features, COMs were generated from the grey-tone images.

\subsection{Software}

Image Processing Tools for Windows $95^{\circledR}$ software was developed at the Instrumentation and Sensing Laboratory using the Microsoft $\mathrm{C}++$ (Microsoft Co., Roselle, IL) compiler. The software provides many basic image processing functions, such as converting image format, cropping the region of interest (ROI) in an image, measuring image size, enhancing linear contrast, resizing, generating image data for texture analysis, and analyzing multispectral images.

The size of an original spectral image captured by a camera was $786 \times 493(387$, 498 pixels). This image was binned to reduce its size to $254 \times 240$ (60,960 pixels). The intensity of each pixel in the new image was an average of a $3 \times 2$ binning from an original image. Finally, a $64 \times 64$ subimage was cropped from the reduced images for fast processing of the COM. The image was first loaded into an active window. The centroid (point on the image) was calculated from the ROI to generate subimages from the body of the chicken as shown in Fig. 1. These subimages were used for further texture analysis

\subsection{Co-occurrence matrix of image texture}

A co-occurrence matrix contains not only texture information but spectral information as well. Haralick et al. (1973) presented the general procedure for extracting texture properties from image data in the spatial domain. They computed 
a set of grey-tone spatial-dependence probability-distribution matrices for a given image and demonstrated that texture features can be extracted from each COM.

COMs measure the probability that a pixel of a particular grey level occurs at an orientation and a specified distance from its neighboring pixels given that these pixels have a particular grey level. A COM is represented by the function $P(i, j, d$, $\theta$ ), where $i$ represents the grey level at coordinate $(x, y)$, and $j$ represents the grey level of its neighbor pixel at a distance $d$ and an orientation $\theta$ from location $(x, y)$. The eight nearest-neighbor resolution cells $(3 \times 3$ matrix $)$, or surrounding resolution cells, are expressed in terms of their spatial orientation to the central reference pixel $(i, j)$. The eight neighbors represent all image pixels at a distance of 1 . For instance, resolution cells $(i+1, j)$ and $(i-1, j)$ are nearest-neighbors to the reference cell $(i$, $j$ ) in the horizontal direction $\left(\theta=0^{\circ}\right)$ and at a distance, $d=1$ as shown in Fig. 2. This concept was extended to the three additional orientations $(\theta=45,90$, and $135^{\circ}$ ) for the performance of texture feature analysis (Park and Chen, 1996).

A COM is scale invariant, i.e. the matrix would be the same if all pixels of a certain grey level in an image were changed by a constant value. These matrices show the relative frequency distributions of grey levels and describe how often one grey level will appear in a specified spatial relationship to another grey level for each image region. Since there are eight nearest neighbors for each pixel, many different COMs can be derived from the same grey-tone image, based on direction and distance from the reference cell for each image region. In this study, the

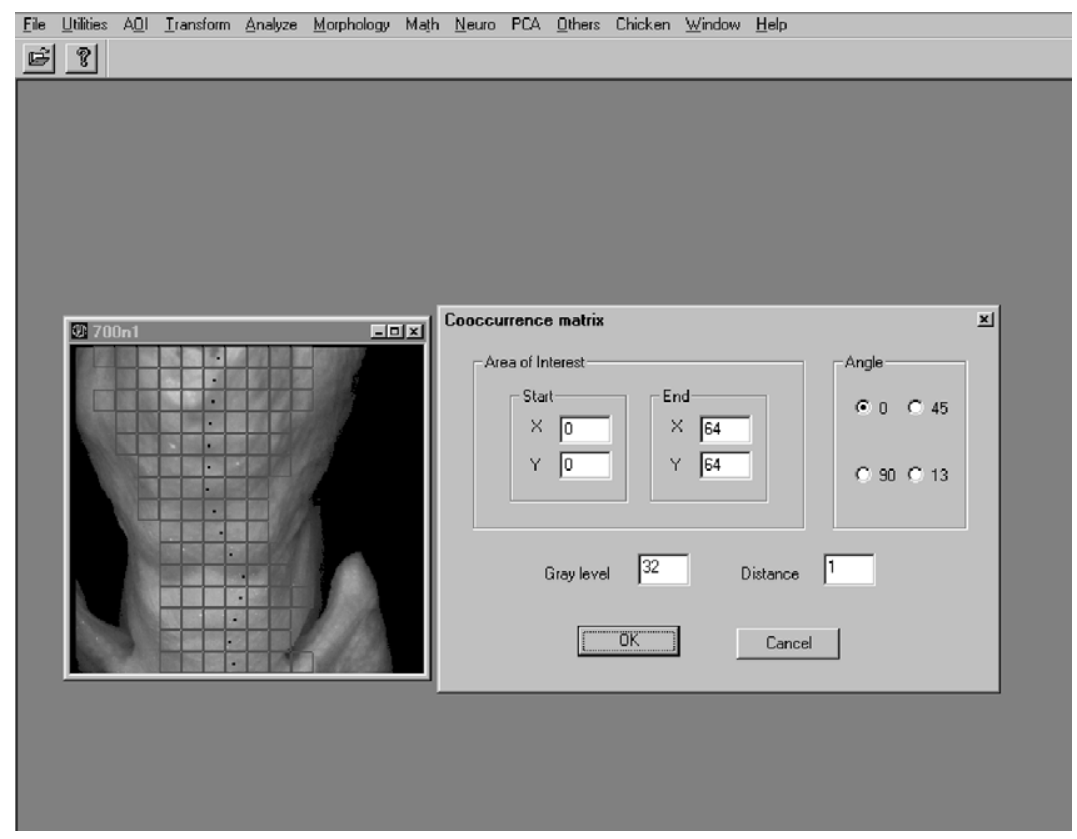

Fig. 1. Chicken spectral image and its subimages generated by centroid for further co-occurrence matrix texture analysis. 

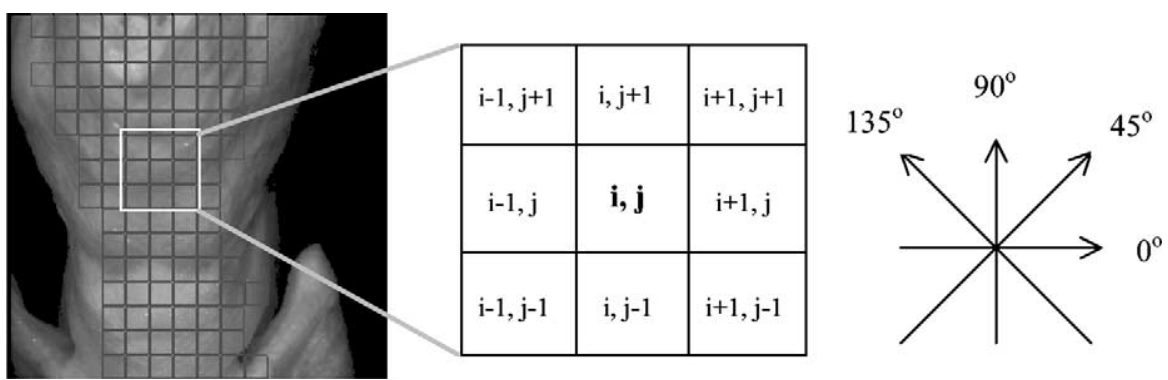

Fig. 2. Nearest-neighbour resolution cell diagram for texture analysis; $(i, j)$, resolution cell coordinates from a typical spectral image of poultry carcass.

textural features were calculated from the COMs for direction $(\theta)$ equals $0,45,90$, and $135^{\circ}$ and distance $(d)$ equals 1 and 2, respectively. For further details concerning COMs, see Park and Chen (1996).

\subsection{Texture features}

Due to their statistical significance in the classification model, the following features were used to extract data from the multispectral images for use in classification models. The angular second moment feature is a measure of image homogeneity and is calculated from the normalized COM. Higher values of this feature correspond to lower amplitude or small intensity changes in the image, which produce a sparse co-occurrence matrix. The contrast feature measures local variations in an image. Higher contrast values indicate high amounts of local variation. The correlation feature is a measure of uniformity of intensity values in an image. For an image with large areas of similar intensities, correlation will be much higher than for an image with noisier, uncorrelated intensities.

Other textural features examined in this study were sum average, sum variance, sum entropy, entropy, difference variance, and difference entropy. Sum average and sum variance features are the average and variance of the normalized grey-tone image in the spatial domain, respectively. The sum entropy feature is a measure of randomness within an image. Entropy is an indication of the complexity within an image. Thus more complex images have higher entropy values. Both difference variance and difference entropy, which are calculated from a normalized COM, indicate the amount of randomness in an image. The formula for calculating texture features described above have been mathematically explained in detail elsewhere (Haralick et al., 1973).

\subsection{Discriminant analysis}

Discriminant analysis is useful for predicting to which class an observation belongs, based on knowledge of the quantitative variables and a set of linear combinations of those variables. Linear or quadratic discriminant functions can be 
used for data with approximately multivariate normal distributions within classes. The performance of a discriminant function can be evaluated by estimating error rates (probabilities of misclassification), which can be estimated by cross-validation (SAS, 1990). Leave-one-out cross-validation uses $(n-1)$ samples out of $(n)$ observations as a training set. It determines the discriminant functions based on these $(n-1)$ observations and then applies them to classify the one observation left out. This was done for each of the $(n)$ observations. The misclassification rate for each class was obtained based on the proportion of sample observations in the group that were misclassified.

Discriminant functions classify observations into two or more groups on the basis of quantitative variables. When the distribution within each group is assumed to be multivariate normal, a parametric method can be used to derive a linear or quadratic discriminant function. In most cases, the discriminant function is determined by a measure of generalized squared distance. The classification criterion can be based on either the individual within-group covariance matrices for the quadratic function, or the pooled covariance matrix for the linear function. Each observation is placed in the class from which it has the smallest generalized squared distance. This method also computes the posterior probability of an observation belonging to each class.

\section{Results and discussion}

\subsection{Variability of COM textural feature}

Texture features varied with the distance and angle of the COM, as well as condemnation of the poultry carcasses. Texture features between wholesome and unwholesome carcasses were compared at all six wavelengths based on the statistical significance $t$-test. However, only the results from the two most significant wavelengths (542 and $700 \mathrm{~nm}$ ) are reported. Table 1 shows significance test results for comparing mean values of texture features to distinguish between wholesome and unwholesome carcasses for images at 542 and $700 \mathrm{~nm}$. Angular second moment, variance, sum average, sum variance, and sum entropy were more significant features, which could be used as input variables for model development.

Feature values of the wholesome carcasses scanned at $542 \mathrm{~nm}$, were significantly different from unwholesome carcasses with septicemic $(P \leq 0.005)$ and cadaver $(P \leq 0.005)$ conditions, but no significant difference was found between septicemic and cadaver carcasses for the COM with a distance of 1 pixel and an angle of 0 . However, the texture features of septicemic carcasses $\left(d=1\right.$ pixel and $\left.\theta=0^{\circ}\right)$ were significantly $(P \leq 0.01)$ different from those of cadaver carcasses $(d=2$ pixel and $\left.\theta=0^{\circ}\right)$. The feature values of septicemic and cadaver carcasses were significantly $(P \leq 0.005)$ different from the features of wholesome carcasses when distances equal 1 or 2 pixels and the angles equal $0,45,90$, or $135^{\circ}$. Within the same carcass groups, the texture feature values at $\theta=0^{\circ}$ were much higher $(P \leq 0.005)$ than the feature values at $\theta=45,90$, and $135^{\circ}$. Thus, the characteristics of textural features 
were affected by not only unwholesomeness of chickens, but also the parameters of COM, specifically distance and angle. Therefore, optimum parameter values (in this case, $d=1$ pixel and $\theta=0^{\circ}$ ) need to be selected for classifying wholesome carcasses from unwholesome carcasses with high classification accuracy.

For the carcasses scanned at $700 \mathrm{~nm}$, the feature values of septicemic and cadaver carcasses $\left(d=1\right.$ pixel and $\left.\theta=0^{\circ}\right)$ were significantly $(P \leq 0.005)$ different from the feature values of wholesome carcasses $\left(d=2\right.$ pixel and $\theta=45,90$, and $\left.135^{\circ}\right)$. However, no significant difference was found between septicemic and cadaver carcasses with a distance of 1 and an angle of $0^{\circ}$. Therefore, the texture features obtained from COMs with distance 1 and angle $0^{\circ}$ were only found useful for identifying wholesome poultry carcasses.

Fig. 3 compares the feature values of mean angular second moment from COMs at 542 and $700 \mathrm{~nm}$ as a function of distance and angle for three different carcasses (normal, septicemia, and cadaver). Angular second moment of the wholesome carcasses scanned at $542 \mathrm{~nm}$ was lower than that of septicemic $(P \leq 0.005)$ and cadaver $(P \leq 0.005)$ carcasses, but no significant difference was found between septicemic and cadaver carcasses when the COMs with a distance of 1 or 2 pixels and an angle of $0,45,90$, or $135^{\circ}$ were compared (Fig. 3a). Similarly, the mean values of the angular second moment feature obtained from the spectral images of wholesome carcasses scanned at $700 \mathrm{~nm}$ was lower than those of septicemic $(P \leq 0.05)$ and cadaver $(P \leq 0.05)$ carcasses. However, the value of septicemic carcasses was lower than that of cadaver carcasses at all different texture parameters $\left(d=1\right.$ and 2 pixels; $\theta=0,45,90$, and $\left.135^{\circ}\right)$. As shown in Fig. 3b, the mean angular second moment values of wholesome, septicemic and cadaver carcass $(d=1$

Table 1

$T$-test ( $P$-values) results for comparing mean values between wholesome and unwholesome carcasses based on textural features of COMs for dual-wavelength images

Texture features of co-occurrence matrix

Wavelength (nm)

542

700

Angular second moment

Contrast

Correlation

Variance

Inverse difference moment

Sum average

Sum variance

Sum entropy

Entropy

Difference variance

Difference entropy

Information measures of correlation-1

Information measures of correlation-2

$$
\begin{aligned}
&-2.929(0.001) \\
&-0.847(0.412) \\
& 1.631(0.125) \\
&-23.743(0.0001) \\
&-0.283(0.782) \\
& 38.304(0.0001) \\
& 23.446(0.0001) \\
& 6.192(0.0001) \\
& 1.446(0.170) \\
&-0.292(0.775) \\
& 0.137(0.893) \\
&-0.411(0.688) \\
& 1.038(0.317)
\end{aligned}
$$

Wholesome $(n=73)$ and unwholesome $(n=103)$ including septicemia $(n=30)$, cadaver $(n=48)$, tumor $(n=16)$, skin-tear $(n=5)$, and bruise $(n=4)$ carcasses were used for the experiments. 


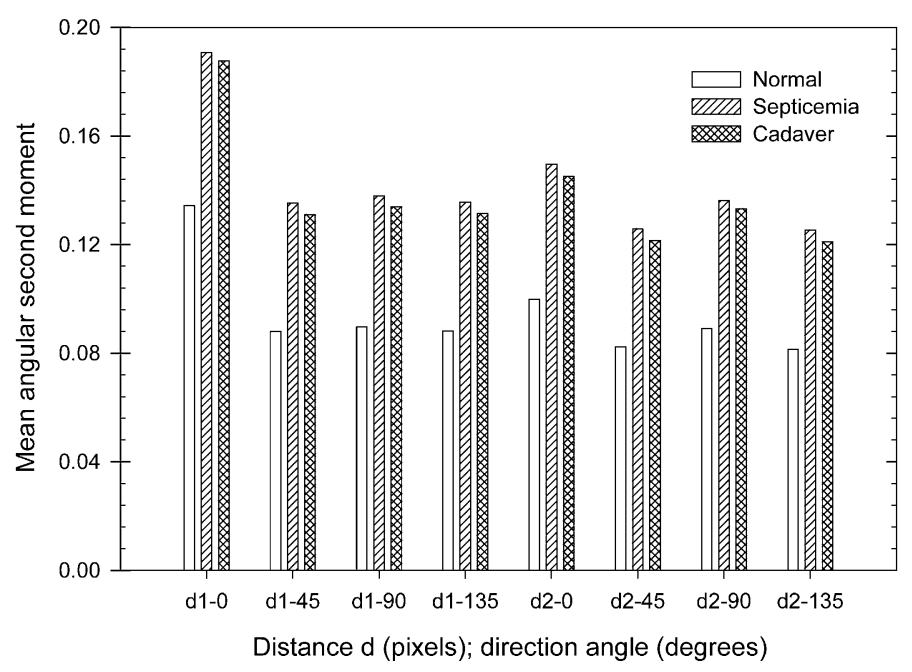

(a) $542 \mathrm{~nm}$

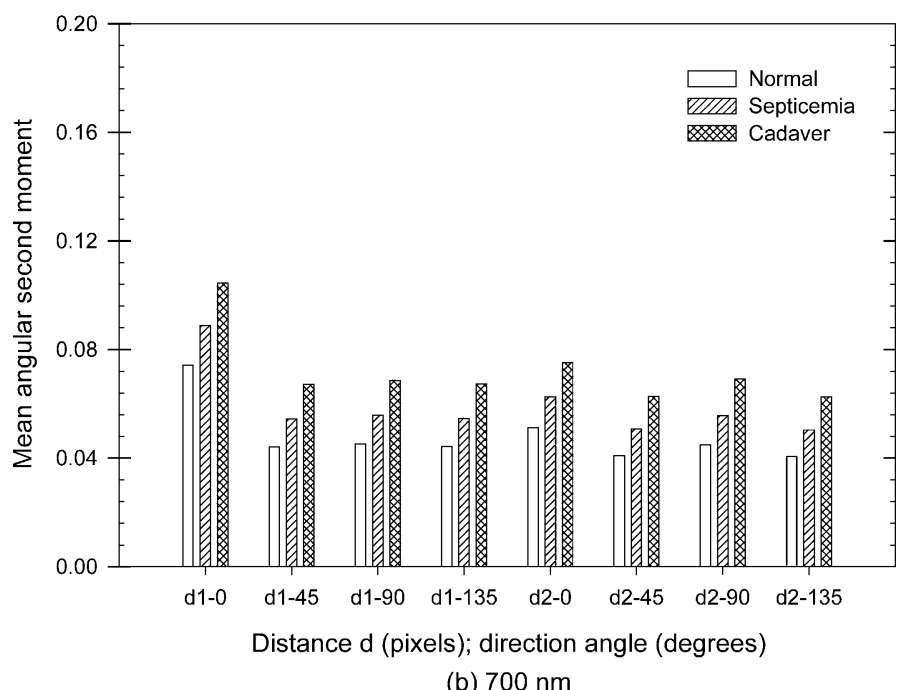

Fig. 3. Mean angular second moment from co-occurrence matrix at wavelengths (a) $542 \mathrm{~nm}$ and (b) 700 $\mathrm{nm}$ from normal, septicemic, and cadaver poultry carcasses.

pixel and $\left.\theta=0^{\circ}\right)$ was higher $(P \leq 0.005)$ than those values at $\theta=45,90$, and $135^{\circ}$ with a distance of 1 or 2 pixels. Thus, the texture feature values of spectral images changed with the parameters of distance and angle (or orientation). The results show that COM data analysis enables us to identify individual condemned carcasses such as septicemia and cadaver conditions. 


\subsection{Accuracy of discriminant model}

Discriminant models at the selected wavelengths were developed to separate unwholesome carcasses from wholesome carcasses. All models were calibrated and validated by the cross-validation method (Table 2). The accuracy of the discriminant models varied not only with the parameters for generating the COMs, but also with the linearity of the models. For poultry inspection applications, linear discriminant models were adequate for the classification of unwholesome carcasses at both 542 and $700 \mathrm{~nm}$ for a distance of 1 pixel.

The average accuracy of the linear discriminant model for identifying unwholesome poultry carcasses was $90.5 \%$ when accuracy values at both 542 and $700 \mathrm{~nm}$ were included. Since the average accuracy for identifying wholesome carcasses was low (less than 51.5\%), linear models have a greater difficulty predicting wholesome poultry carcasses.

The quadratic model had an average accuracy of about $75 \%$ for identifying wholesome carcasses, which was independent of co-occurrence matrix orientation. However, the overall accuracy of identifying unwholesome carcasses was $62 \%$, which exhibited low classification accuracies at all angle orientations. Therefore, the quadratic models are less accurate than the linear models for poultry carcasses classification using a COM distance of 1 pixel.

Similar results were obtained (Table 3 ) when the distance was 2 pixels for both linear and quadratic discriminant models. The linear models classified unwholesome carcasses with high accuracy no matter which orientation parameters were applied. However, similar to the models for $d=1$ pixel, average classification accuracy for wholesome carcasses was poor $(56.1 \%)$. Compared with the linear models, the quadratic models classified both wholesome and unwholesome carcasses with much lower accuracy.

Table 2

Classification accuracy of wholesome and unwholesome carcasses through statistical discriminant algorithm with co-occurrence matrix textural data with orientations of 0,45 , 90, and $135^{\circ}$ and a distance of 1

\begin{tabular}{|c|c|c|c|c|c|c|c|c|c|}
\hline \multirow[t]{3}{*}{ Wavelength (nm) } & \multirow[t]{3}{*}{ Class } & \multicolumn{8}{|c|}{ Model } \\
\hline & & \multicolumn{4}{|c|}{ Linear } & \multicolumn{4}{|c|}{ Quadratic } \\
\hline & & $0^{\circ}$ & $45^{\circ}$ & $90^{\circ}$ & $135^{\circ}$ & $0^{\circ}$ & $45^{\circ}$ & $90^{\circ}$ & $135^{\circ}$ \\
\hline \multirow[t]{2}{*}{542} & UW & 90.3 & 91.4 & 90.3 & 91.4 & 60.2 & 62.4 & 62.4 & 62.4 \\
\hline & $\mathrm{W}$ & 45.5 & 51.5 & 51.5 & 51.5 & 78.8 & 72.7 & 75.8 & 72.7 \\
\hline \multirow[t]{2}{*}{700} & UW & 87.1 & 90.3 & 91.4 & 91.4 & 76.3 & 76.3 & 67.7 & 62.4 \\
\hline & $\mathrm{W}$ & 47.1 & 44.1 & 38.2 & 44.1 & 64.7 & 58.8 & 70.6 & 64.7 \\
\hline
\end{tabular}

Sample numbers are calibration and validation $(n=176)$. Cross-validation method was used for the validation of the models. Unwholesome carcasses include septicemia, cadaver, tumor, skin-tear, and bruise. W, wholesome; UW, unwholesome. 
Table 3

Classification accuracy of wholesome and unwholesome carcasses through statistical discriminant algorithm with co-occurrence matrix textural data with orientations of 0,45 , 90, and $135^{\circ}$ and a distance of 2

\begin{tabular}{|c|c|c|c|c|c|c|c|c|c|}
\hline \multirow[t]{3}{*}{ Wavelength (nm) } & \multirow[t]{3}{*}{ Class } & \multicolumn{8}{|c|}{ Model } \\
\hline & & \multicolumn{4}{|c|}{ Linear } & \multicolumn{4}{|c|}{ Quadratic } \\
\hline & & $0^{\circ}$ & $45^{\circ}$ & $90^{\circ}$ & $135^{\circ}$ & $0^{\circ}$ & $45^{\circ}$ & $90^{\circ}$ & $135^{\circ}$ \\
\hline \multirow[t]{2}{*}{542} & UW & 89.3 & 90.3 & 91.4 & 92.5 & 68.8 & 66.9 & 53.8 & 54.8 \\
\hline & $\mathrm{W}$ & 54.6 & 54.6 & 57.6 & 57.6 & 75.8 & 75.8 & 78.8 & 72.7 \\
\hline \multirow[t]{2}{*}{700} & UW & 90.3 & 91.4 & 89.3 & 88.2 & 78.5 & 75.3 & 69.9 & 66.7 \\
\hline & $\mathrm{W}$ & 52.9 & 38.2 & 38.2 & 38.2 & 61.8 & 58.8 & 70.6 & 58.8 \\
\hline
\end{tabular}

Sample numbers are calibration and validation $(n=176)$. Cross-validation method was used for validation of the models. Unwholesome carcasses include septicemia, cadaver, tumor, skin-tear, and bruise. W, wholesome; UW, unwholesome.

Table 4 shows the accuracy of discriminant models (using a COM distance of 1) for classifying poultry carcasses into three classes: wholesome, septicemic and cadaver. Overall classification accuracies were lower than the two-class (wholesome and unwholesome) cases. When using 542-nm data, the accuracies of both the linear and quadratic models were best for the cadaver carcass class. The same trend occurred for the linear models using 700-nm data. Overall, the quadratic models performed slightly better than the linear models for identifying wholesome carcasses, however, little improvement was made for individual condemned carcasses such as septicemic and cadaver. The quadratic models using 700-nm spectral images performed best for identifying septicemic carcasses with an accuracy of $60 \%$, but

Table 4

Classification accuracy of wholesome and unwholesome carcasses through statistical discriminant algorithm with co-occurrence matrix textural data with orientations of 0,45 , 90, and $135^{\circ}$ and a distance of 1

\begin{tabular}{|c|c|c|c|c|c|c|c|c|c|}
\hline \multirow[t]{3}{*}{ Wavelength (nm) } & \multirow[t]{3}{*}{ Class } & \multicolumn{8}{|c|}{ Model } \\
\hline & & \multicolumn{4}{|c|}{ Linear } & \multicolumn{4}{|c|}{ Quadratic } \\
\hline & & $0^{\circ}$ & $45^{\circ}$ & $90^{\circ}$ & $135^{\circ}$ & $0^{\circ}$ & $45^{\circ}$ & $90^{\circ}$ & $135^{\circ}$ \\
\hline \multirow[t]{3}{*}{542} & $\mathrm{~W}$ & 51.5 & 63.6 & 60.6 & 60.6 & 75.8 & 75.8 & 75.8 & 72.7 \\
\hline & $\mathrm{S}$ & 23.3 & 26.7 & 23.3 & 30.0 & 13.3 & 26.7 & 30.0 & 23.3 \\
\hline & $\mathrm{C}$ & 79.2 & 87.2 & 79.2 & 81.3 & 77.1 & 83.0 & 83.3 & 81.3 \\
\hline \multirow[t]{3}{*}{700} & $\mathrm{~W}$ & 61.8 & 52.9 & 52.9 & 52.9 & 64.7 & 61.8 & 61.8 & 52.9 \\
\hline & $\mathrm{S}$ & 40.0 & 26.7 & 23.3 & 40.0 & 56.7 & 60.0 & 53.3 & 46.7 \\
\hline & $\mathrm{C}$ & 75.0 & 79.2 & 79.2 & 79.2 & 47.9 & 47.9 & 43.8 & 50.0 \\
\hline
\end{tabular}

Sample numbers are calibration and validation $(n=151)$. Cross-validation method was used for validation of the models. $\mathrm{W}$, wholesome; S, septicemia; $\mathrm{C}$, cadaver. 
Table 5

Classification accuracy of wholesome and unwholesome carcasses through statistical discriminant algorithm with co-occurrence matrix textural data with orientations of $0,45,90$, and $135^{\circ}$ and a distance of 2

\begin{tabular}{|c|c|c|c|c|c|c|c|c|c|}
\hline \multirow[t]{3}{*}{ Wavelength (nm) } & \multirow[t]{3}{*}{ Class } & \multicolumn{8}{|c|}{ MODEL } \\
\hline & & \multicolumn{4}{|c|}{ Linear } & \multicolumn{4}{|c|}{ Quadratic } \\
\hline & & $0^{\circ}$ & $45^{\circ}$ & $90^{\circ}$ & $135^{\circ}$ & $0^{\circ}$ & $45^{\circ}$ & $90^{\circ}$ & $135^{\circ}$ \\
\hline \multirow[t]{3}{*}{542} & $\mathrm{~W}$ & 60.6 & 57.6 & 60.6 & 54.6 & 75.8 & 75.8 & 75.8 & 75.8 \\
\hline & $\mathrm{S}$ & 33.3 & 23.3 & 26.7 & 26.7 & 16.7 & 13.3 & 23.3 & 13.3 \\
\hline & C & 87.5 & 89.6 & 83.3 & 81.3 & 85.4 & 77.1 & 79.2 & 83.3 \\
\hline \multirow[t]{3}{*}{700} & W & 67.7 & 55.9 & 50.0 & 47.1 & 67.7 & 67.7 & 61.8 & 52.9 \\
\hline & $\mathrm{S}$ & 36.7 & 40.0 & 40.0 & 36.7 & 43.3 & 60.0 & 43.3 & 63.3 \\
\hline & C & 75.0 & 79.2 & 66.7 & 81.3 & 37.5 & 45.8 & 39.6 & 52.1 \\
\hline
\end{tabular}

Sample numbers are calibration and validation $(n=151)$. Cross-validation method was used for validation of the models. $\mathrm{W}$, wholesome; $\mathrm{S}$, septicemia; $\mathrm{C}$, cadaver.

performed poorly for cadaver identification (only 47.9\%). Even though the quadratic models classify septicemic carcasses better than the linear models, accuracies are quite low.

For the COMs with a distance of 2 pixels, similar results were obtained (Table 5). Neither linear nor quadratic models successfully classified septicemic carcasses, whereas, cadaver carcasses could be classified by the linear model using $45^{\circ}$ at 542-nm wavelength.

\section{Conclusions}

Discriminant models for classifying poultry carcasses were developed with linear and quadratic covariance matrix analysis methods with the selected COM image texture features. From tests of many discriminant models, no single model could identify both wholesome and unwholesome carcasses with acceptably high classification accuracies (over $90 \%$ for both identification). Also, the classification accuracy depended upon the parameters of the texture features and the spectral image wavelengths. Of the two types of discriminant models tested, linear discriminant models appear to be the most capable for identifying unwholesomeness of carcasses. It remains to be determined whether the classification rate of unwholesomeness and the rate of false positives is acceptable for on-line inspection.

In addition, because texture features of spectral images vary with both spectral and spatial parameters, selection of optimum wavelengths and distance and orientation of COMs are important to obtain good discriminant models for application of dual-wavelength imaging. Further discriminant model development utilizing other parameters selected from textural feature analysis should be conducted to improve the classification accuracy of poultry carcasses. All models reported in this paper 
were obtained from the individual spectral wavelength images. Further spectral image preprocessing with multi-wavelengths, such as combined images from different spectra or image ratios, could be investigated to improve classification accuracy.

\section{References}

Daley, W., Carey, R., Thomson, C., 1994. Real-time color grading and defect detection of food products. SPIE 2345, 403-411.

Giacomelli, G.A., Ling, P.P., Kole, J., 1998. Determining nutrient stress in lettuce plants with machine vision technology. Hort. Technol. 8 (3), 361-365.

Guyer, D.E., Miller, G.E., Schreiber, M.M., Mitchell, O.R., Vanderbelt, V.C., 1986. Machine vision and image processing for plant identification. Trans. ASAE 29 (6), 863-869.

Haralick, R.M., Shanmugam, K., Dinstein, I., 1973. Textural features for image classification. IEEE Trans. Syst. Man Cybern. SMC 3 (6), 610-621.

Liu, Y., Aneshansley, D.J., Stouffer, J.R., 1993. Autocorrelation of ultrasound speckle and its relationship to beef marbling. Trans. ASAE 36 (3), 971-977.

Luo, X., Jayas, D.S., Symons, S.J., 1999. Identification of damaged kernels in wheat using a colour machine vision. J. Cereal Sci. 30 (1), 49-59.

Majumdar, S., Jayas, D.S., 1999. Single-kernel mass determination for grain inspection using machine vision. Appl. Eng. Agric. 15 (4), 357-362.

Meyer, G.E., Troyer, W.W., Fitzgerald, J.B., Paparozzi, E.T., 1992. Leaf nitrogen analysis of poinsettia (Euphorbia Pulcherrima Will D.) using spectral properties in natural and controlled lighting. Appl. Eng. Agric. 8 (5), 715-722.

Ni, B., Paulsen, M.R., Reid, J.F., 1998. Size grading of corn kernels with machine vision. Appl. Eng. Agric. 14 (5), 567-571.

Paliwal, J., Shashidhar, N.S., Jayas, D.S., 1999. Grain kernel identification using kernel signature. Trans. ASAE 42 (6), 1921-1924.

Park, B., Chen, Y.R., 1994. Intensified multispectral image processing for poultry carcasses inspection. Proceedings of the Food Processing Automation Conference III, Orlando, FL, pp. 97-106.

Park, B., Chen, Y.R., 1996b. Multispectral image co-occurrence matrix analysis for poultry carcasses inspection. Trans. ASAE 39 (4), 1485-1491.

Park, B., Thane, B.R., Whittaker, A.D., 1992. Ultrasonic image analysis for beef tenderness. Optics in agriculture and forestry. SPIE 1836, 120-131.

Park, B., Chen, Y.R., Nguyen, M., Hwang, H., 1996a. Characterizing multispectral images of tumorous, bruised, skin-torn, and wholesome poultry carcasses. Trans. ASAE 39 (5), 1933-1941.

Pearson, T.C., Schatzki, T.F., 1998. Machine vision system for automated detection of aflatoxin-contaminated pistachios. J. Agric. Food Chem. 46 (6), 2248-2252.

SAS/STAT User's Guide. 1990. SAS Institute Inc., Cary, NC.

Shearer, S.A., Holmes, R.G., 1990. Plant identification using color co-occurrence matrices. Trans. ASAE 33 (6), 2037-2044.

Tao, Y., Heinemann, P.H., Varghese, Z., Morrow, C.T., Sommer, J.H., 1995. Machine vision for color inspection of potatoes and apples. Trans. ASAE 38 (5), 1555-1561.

Wang, Z., Heinemann, P.H., Walker, P.N., Heuser, C., 1999. Automated micropropagated sugarcane shoot separation by machine vision. Trans. ASAE 42 (1), 247-254.

Zayas, I.Y., Flinn, P.W., 1998. Detection of insects in bulk wheat samples with machine vision. Trans. ASAE 41 (3), 883-888. 